\title{
Metodologia para avaliação da gamificação em jogos
}

\author{
Bruna Nayara M. Lima ${ }^{1}$, Ludimila da Bela Cruz ${ }^{1}$, Sérgio A. Andrade de Freitas ${ }^{1}$ \\ ${ }^{1}$ Faculdade UnB Gama (FGA) - Universidade de Brasília (UnB) \\ Área Especial de Indústria Projeção A, Setor Leste - Gama \\ 72.444-240 - Brasília - DF - Brazil \\ \{bnml, ludimila\}@alunos.unb.br, sergiofreitas@unb.br
}

\begin{abstract}
The use of games and gamified processes are becoming subject of studies all over the world. The new generations are familiar to virtual environments of information and games. However, the educators must be aware if a game fulfills their pedagogical expectations before using it in a classroom. In this context, the paper presents an evaluation methodology for gamification in games. The evaluation results present the motivational aspects and educational indicators existent in the game.
\end{abstract}

Resumo. A utilização de jogos e processos gamificados na educação tem se tornado um foco de pesquisa em diversas áreas. As novas gerações têm uma vivência natural no ambiente virtual de informação e jogos que a internet proporciona. Entretanto o educador, antes de usar um determinado jogo, deve atentar se este irá ou não atender as suas expectativas pedagógicas. Neste cenário, este artigo apresenta uma proposta de metodologia de avaliação da gamificação em jogos. Como resultados da avaliação são apresentados ao educador as características motivacionais e indicadores educacionais presentes no jogo avaliado.

\section{Introdução}

O uso de jogos como ferramentas de ensino têm se tornado cada vez mais constante. As novas gerações têm feito um uso intensivo das tecnologias digitais modernas, tanto em termos de dispositivos: computadores, celulares e vídeo games, quanto de aplicativos e jogos (McGonigal, 2011; Kumar \& Herger, 2013). Esta geração é nativamente digital (Prensky, 2007) e tem uma "aptidão nata" para aprender interagindo (McGonigal, 2011). O uso de Gamificação na educação desta geração figura uma opção natural, desde o ensino fundamental até o ensino superior.

Entretanto fica a pergunta se todo aplicativo e, em especial, os jogos motivam adequadamente um jogador e produz um engajamento alinhado com critérios desejáveis no processo de ensino e aprendizagem. Algumas referências da área (McGonigal, 2011; Kumar \& Herger, 2013; Appelo, 2014; Kapp, Blair, \& Mesch, 2014) sugerem o uso de jogos como metodologia para o engajamento e motivação dos usuários sem descreverem formalmente quais são os critérios para uma boa gamificação. 
Neste cenário, a introdução de critérios para a avaliação da gamificação quanto aos seus critérios motivacionais e em termos de engajamento do jogador nas atividades pedagógicas introduzidas pelo uso de jogos na educação é altamente desejável. O objetivo deste trabalho é apresentar e testar uma proposta de metodologia de avaliação da gamificação de jogos educacionais pautada na proposta no framework Octalysis proposto por Yu Kai-Chou (Chou, 2015), estendendo-a com: (1) os critérios de engajamento de Fredericks et al (Fredricks, Blumenfeld, \& Paris, 2004) e (2) a criação de uma estrutura interna que permita a rápida avaliação do jogo por parte de um avaliador.

No Brasil, Seixas et al (Seixas, Gomes, Filho, \& Rodrigues, 2014) apresentam um estudo de caso em uma escola do ensino fundamental, fazendo uma análise do engajamento dos estudantes numa gamificação. Outros trabalhos apresentam uma coletânea de trabalhos sobre gamificação e educação no país (Figueiredo, Paz, \& Junqueira, 2015; Borges, Reis, Durelli, Bittencourt, Jaques, \& Isotani, 2013) e propostas de uso de metodologias ativas de aprendizagem (Freitas, Silva, \& Marsicano, 2016).

Esta pesquisa exploratória e teórica está assim estruturada: na seção 2 é apresentado o referencial teórico utilizado, na seção 3 o Octalysis é estendido com a criação de uma estrutura interna para as técnicas de gamificação e respectivas motivações básicas, na seção 4 é apresentada a heurística que realiza a avaliação do jogo a partir da estrutura interna proposta, na seção 5 é avaliada a metodologia proposta através da sua aplicação no jogo "Advise to the King" e, por fim, na seção 6 são apresentadas algumas conclusões e trabalhos futuros.

\section{Referencial Teórico}

De acordo com McGonigal (McGonigal, 2011) existem quatro características que determinam um jogo: meta, regras, feedback e participação voluntária. A meta indica o objetivo pelo qual um jogador deseja jogar o jogo. As regras indicam as normas, restrições e ações permitidas que norteiam o jogador no seu percurso dentro do jogo. O feedback são as respostas do jogo a cada ação executada, indicando o quanto o jogador está distante ou não da meta do jogo. Já a participação voluntária é a liberdade que o jogador tem de tentar ou não o jogo.

Tomando por base o roteiro proposto por McGonigal (McGonigal, 2011), fica evidente que em todas as fases da gamificação é necessário avaliar a motivação do jogador no jogo. O framework Octalysis proposto por Yu Kai-Chou (Chou, 2015) apresenta oito motivações básicas (MB) que induzem os jogadores a jogarem e elenca 74 técnicas de jogos (game techniques) distribuídas nas motivações básicas. As motivações básicas são:

1. Significado Épico \& Chamado - quando o jogador acredita que está fazendo algo maior, por um bem maior ou que foi "escolhido" para fazer alguma coisa transcendental.

2. Desenvolvimento \& Realização - quando o jogador observa seus avanços, progressos, desenvolvimento de habilidades e, eventualmente, a superação de desafios.

3. Empoderamento \& Feedback - quando o jogador está envolvido num processo criativo onde repetidamente tem de descobrir algo e tentar combinações diferentes. 
V Congresso Brasileiro de Informática na Educação (CBIE 2016)

Anais do XXVII Simpósio Brasileiro de Informática na Educação (SBIE 2016)

4. Propriedade \& Posse - quando o jogador é motivado porque tem a sensação de posse ou propriedade de algo.

5. Influência Social \& Pertencimento - quando o jogador é motivado por elementos sociais que influenciam as pessoas, incluindo: orientação, aceitação, respostas sociais, companheirismo, bem como a competição e a inveja.

6. Escassez \& Impaciência - quando o jogador é motivado pelo desejo de algo que não pode ter.

7. Imprevisibilidade \& Curiosidade - quando o jogador é motivado por querer descobrir o que vai acontecer a seguir. Se ele não sabe o que vai acontecer, o seu cérebro está envolvido e pensa sobre isso muitas vezes.

8. Perda \& Rejeição - quando o jogador é motivado pela prevenção de algo negativo que possa ocorrer.

Para cada uma das motivações básicas existe um conjunto de técnicas associadas. Alguns exemplos são: as técnicas Narrativa (\#10), Sorte de principiante (\#23) e Elitismo (\#26) associadas a motivação básica "Significado Épico \& Chamado", Barra de progresso (\#4), Pontos (\#1) e Medalhas (\#2) associadas a motivação básica "Desenvolvimento \& Realização". Os números entre parênteses indicam uma referência numérica da técnica.

Para Chou (Chou, 2015), um jogador com um determinado perfil: competitivo, colaborativo, ansioso, solidário entre outros, está em sintonia com um conjunto de motivações através da escolha das técnicas apropriadas. Ao se utilizar técnicas que fortaleçam as motivações correspondentes, haverá um forte engajamento do jogador com a produção de uma sensação de atividades gratificantes e recompensadoras.

\section{Estruturação interna das técnicas de gamificação}

No framework Octalysis de Chou (Chou, 2015) cada técnica representa um elemento de jogo e é apresentada de forma descritiva. Entretanto não há uma definição clara das características que devem estar presentes para que uma técnica seja considerada elemento de gamificação. Falta a definição de um conjunto mínimo de atributos que descrevam formalmente as características particulares de cada técnica.

A solução aqui proposta foi a criação de uma estrutura interna composta por indicadores e valores (par atributo-valor) que caracterizem cada técnica e, ao mesmo tempo, evidenciem as características de cada motivação básica composta por um conjunto de técnicas. Para a escolha dos atributos, foi feito um estudo nos indicadores de engajamento definidos por Fredericks et al (Fredricks, Blumenfeld, \& Paris, 2004). Fredericks et al apresentam três tipos de engajamento, o engajamento emocional, o comportamental e o cognitivo, cada tipo de engajamento possui diferentes indicadores.

Nesta fase do trabalho, foram selecionados indicadores com forte aderência a dois requisitos: ser um atributo que indique o engajamento do aluno nas atividades pedagógicas e ser um atributo que expresse o engajamento do jogador nas atividades do jogo. Os indicadores selecionados para compor as técnicas de gamificação são: envolvimento com o trabalho, participação, atenção, persistência, domínio e socialização. 
V Congresso Brasileiro de Informática na Educação (CBIE 2016)

Anais do XXVII Simpósio Brasileiro de Informática na Educação (SBIE 2016)

Além de compor a estrutura das técnicas, os indicadores servem de espelho para o envolvimento do usuário com a técnica de gamificação. Como as técnicas e as motivações básicas estão ligadas, os indicadores acabam por exercer influência sobre as MBs e podem ser utilizadas como critério de avaliação de gamificação.

Cada indicador recebe um valor que serve de medida de aderência à técnica de gamificação. A escala de valores é composta de cinco valores que variam de um a cinco (escala de Likert): uma nota 1 (um) aponta que o indicador quase não tem influência na técnica de gamificação, uma nota 2 (dois) indica que a influência do atributo é fraca sobre a técnica, uma nota 3 (três) aponta uma influência suficiente do atributo sobre a técnica de gamificação, uma nota (4) quatro indica uma forte influência do atributo na técnica e, finalmente, uma nota cinco aponta uma influência plena do atributo sobre a utilização da técnica de gamificação. A Figura 1 exemplifica a estrutura interna para algumas das técnicas da motivação básica "Significado Épico \& Chamado".

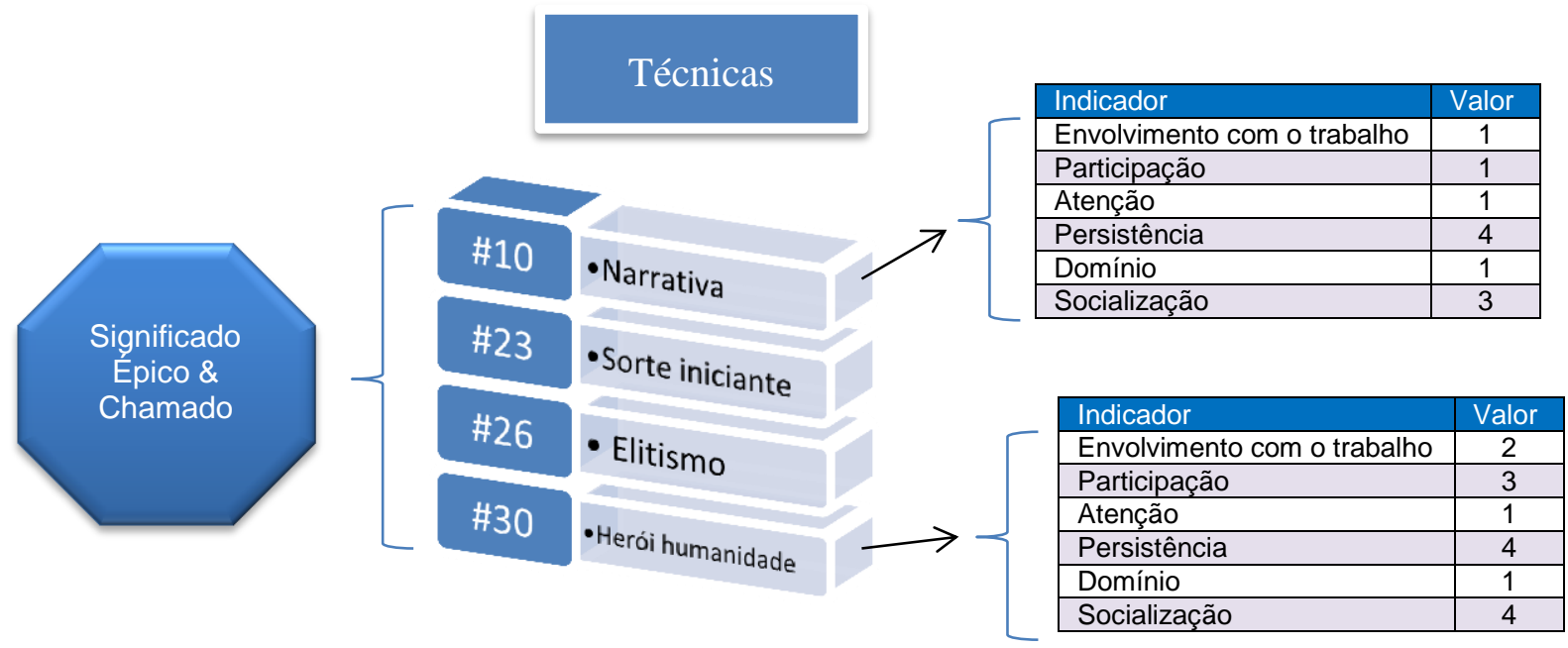

Figura 1: Estrutura interna das técnicas de gamificação.

A Figura 1 apresenta a composição das motivações básicas, das técnicas de gamificação e dos indicadores de engajamento. O octógono à esquerda representa uma das motivações básicas, como exemplo foi utilizado o significado épico e chamado. A tabela central indica algumas das técnicas que compõe a MB. E, finalmente, as tabelas à direita apresentam os indicadores de engajamento e o valor de cada indicador na respectiva técnica. As MBs são compostas por um número variado de técnicas de gamificação, porém as técnicas possuem um número fixo de indicadores.

Para o cálculo dos valores dos atributos, foram utilizados três avaliadores com conhecimento das técnicas de gamificação e do emprego educacional dos atributos selecionados. O valor final dos atributos de cada técnica é a média aritmética do valor atribuído por cada avaliador. $\mathrm{O}$ processo de avaliação foi blind. $\mathrm{O}$ desvio padrão médio total foi de 0,74 no total das 1332 avaliações realizadas (74 técnicas, 6 atributos e 3 avaliadores). $\mathrm{O}$ que denota um conhecimento bem alinhado dos avaliadores. 
V Congresso Brasileiro de Informática na Educação (CBIE 2016)

Anais do XXVII Simpósio Brasileiro de Informática na Educação (SBIE 2016)

As técnicas representam os elementos de jogo que compõem a parte do framework responsável por colocar a gamificação em prática. Os indicadores avaliados mensuram o envolvimento do jogador com as técnicas e, por conseguinte, com as motivações básicas e com a gamificação em si. Com a estrutura interna definida e valorada é possível a proposição de uma metodologia de avaliação da gamificação de um dado jogo.

\section{A metodologia para avaliação dos jogos}

Considerando a estruturação das técnicas, sua projeção sobre as motivações básicas e, principalmente, os indicadores que valoram cada uma das técnicas foi elaborada uma heurística que avalia a gamificação do jogo. Nela um avaliador externo com conhecimentos sobre o jogo a ser avaliado e com conhecimento mediano das técnicas de jogos (mediante um tutorial) é a fonte primária das informações.

O resultado da heurística de avaliação é a identificação das motivações básicas predominantes e dos atributos mais fortemente estimulados pela gamificação avaliada. Com este resultado o avaliador ou outra pessoa qualquer pode, por exemplo, comparar as características próprias de um dado jogo com as características desejadas ao se utilizar um dado jogo. A seguir são detalhados os tipos de avaliação e as etapas que compõem a heurística.

\subsection{Tipos de avaliação}

São três os tipos de avaliação calculados pela heurística: Avaliação simples, avaliação completa e avaliação profunda. A Figura 2 ilustra estes três tipos, bem como a relação entre as entradas e saída de cada uma delas.

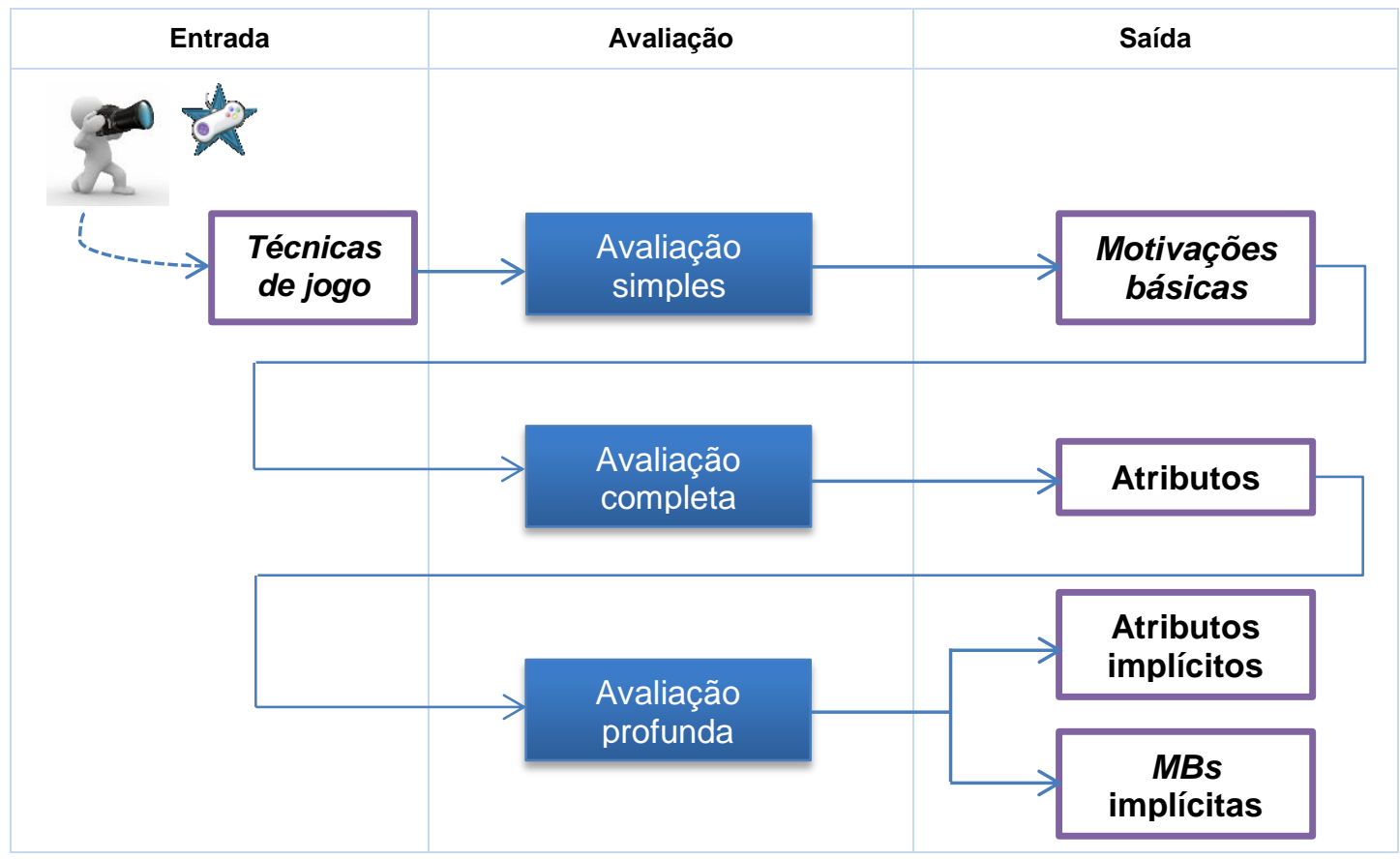

Figura 2: Tipos de avaliações. 
V Congresso Brasileiro de Informática na Educação (CBIE 2016)

Anais do XXVII Simpósio Brasileiro de Informática na Educação (SBIE 2016)

A avaliação é iniciada a partir das técnicas presentes no jogo. Para que então possam ser identificadas as motivações básicas presentes no jogo. A escolha das técnicas como ponto de partida da avaliação se deu pela maior tangibilidade das técnicas em comparação às motivações, caso o avaliador não seja especialista em gamificação e nem no framework utilizado como base da avaliação.

A avaliação simples tem como objetivo identificar quais são a motivações básicas presentes no jogo. Ela tem como entrada a indicação, pelo avaliador, das técnicas existentes no jogo a ser avaliado. Tipicamente o avaliador deve jogar o jogo e constatar a existência ou não de uma dada técnica. A saída são as motivações básicas identificadas e classificadas de acordo com sua relevância no jogo.

Avaliação completa tem como objetivo identificar quais são os indicadores que mais influenciam as motivações básicas encontradas. Ela tem como entrada o conjunto de motivações básicas previamente identificadas e classificadas, bem como o conjunto de técnicas elencadas pelo avaliador. A saída é o conjunto dos indicadores valorados. Este conjunto permite identificar o quanto cada atributo: envolvimento com o trabalho, participação, atenção, persistência, domínio e socialização, é influenciado pelo jogo.

Por fim, a avaliação profunda tem como objetivo identificar quais são as motivações básicas e os indicadores, ambos implícitos, presentes no jogo. Estas características são consideradas implícitas em virtude de não derivarem diretamente das técnicas de jogos usadas como entrada, mas sim do relacionamento intrínseca existente entre as técnicas de jogos. Ela tem como entrada o conjunto de motivações básicas previamente identificadas e classificadas, o conjunto de atributos previamente identificados e classificados, bem como o conjunto de técnicas elencadas pelo avaliador. A saída é o conjunto de atributos e motivações básicas existentes no jogo.

\subsection{As etapas da metodologia de avaliação}

Considerando os três tipos de avaliação são aqui apresentados o processo de execução da análise em cada uma das etapas.

Ao iniciar a avaliação, são selecionadas as técnicas presentes no jogo (Figura 3). A primeira etapa da avaliação é a seleção das técnicas presentes no jogo. Ela é iniciada enquanto o avaliador ainda está jogando o jogo. Em seguida, as técnicas presentes no jogo podem ser selecionadas a partir da lista de técnicas presentes na estrutura interna apresentada. Até que a seleção seja dada por completa, o avaliador talvez precise recorrer ao jogo frequentemente para reavaliar as técnicas em caso de dúvidas. Durante a seleção das técnicas presentes no jogo, o avaliador passa pela lista de técnicas mapeadas dentro do modelo apresentado e indicam quais identificou. As técnicas presentes são utilizadas como material de trabalho para a identificação das MBs.

Após a conclusão da etapa de seleção de técnicas, as MBs impactadas pelo jogo são identificadas. Para que elas sejam obtidas, o avaliador deve passar por cada uma das técnicas elencadas e, a partir do mapeamento, identificar cada uma das motivações básicas associadas. Para cada MB é computada o número de técnicas utilizadas. Ao final, a lista resultante é classificada por número de técnicas e as três MBs melhor classificadas são 
V Congresso Brasileiro de Informática na Educação (CBIE 2016)

Anais do XXVII Simpósio Brasileiro de Informática na Educação (SBIE 2016)

consideradas como as três mais influentes no jogo. Caso seja uma avaliação simples, o processo de avaliação está finalizado. O conjunto de MBs compõe o resultado da avaliação simples, juntamente com a explicação dos impactos de cada um deles em um jogo.

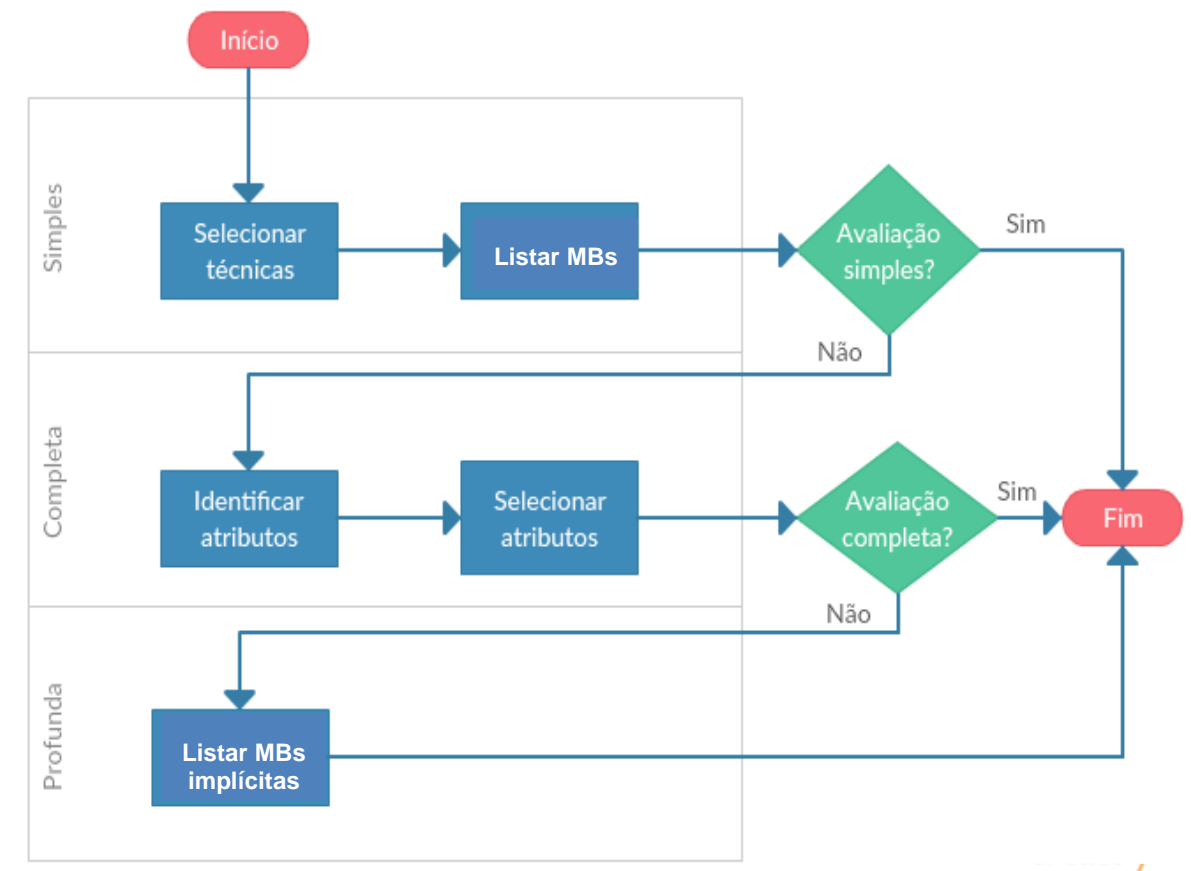

Figura 3: Etapas da avaliação.

A avaliação completa ocorre de forma análoga à avaliação simples. $\mathrm{O}$ conjunto de técnicas presentes no jogo e as motivações básicas impactadas são utilizados como insumo da avaliação. A partir deles, são identificados os atributos que podem ser influenciados a partir da interação com o jogo. Todas as técnicas estão relacionadas em algum nível com os atributos mencionados. Os atributos são analisados de forma a refletirem a participação das técnicas dentro jogo.

Os valores finais $(V F)$ de cada atributo $j$ dentro do jogo são computados através da relação que cada atributo tem com cada uma das técnicas, de acordo com a equação (1).

$$
V F_{j}=\sum_{i=1}^{n} \frac{A t_{i}}{n}
$$

Onde, $V F_{j}$ representa o valor final da técnica, $n$ é a quantidade de técnicas presentes no jogo, $A t_{i}$ do valor do atributo atribuído a cada técnica, dividido pelo número de técnicas e Ati é o valor resultante de cada atributo é extraído da média do montante de cada atributo por técnica presente no jogo. O resultado é uma lista de atributos e valores. Os dois atributos com maior valor são considerado como os mais influenciados pelo jogo e como características mais marcantes deste. 
A avaliação profunda relaciona as motivações básicas diretamente com os atributos mapeados. Por ser uma relação indireta mediada pelas técnicas que interagem diretamente com cada um deles, os resultados são considerados implícitos. A partir das relações indiretas computadas através da identificação de padrões estatísticos são identificadas as motivações básicas impactadas, também de forma indireta.

\section{Avaliação da metodologia}

Para avaliar a metodologia, foi utilizado o jogo "Advisor to the king" (AttK) ${ }^{1}$ de Britt et al (Britt, Hastings, Durik, \& Kopp, 2009). O jogo, Conselheiro do Rei - em tradução livre, foi projetado para ensinar habilidades argumentativas. O jogo foi baseado em um sistema de tutoria de argumentação e em um sistema de feedback inteligente para referências. Attk foi idealizado de forma a integrar o jogador na fantasia desenvolvida para o jogo. O jogador é apresentado como novo funcionário do rei, que deve avaliar petições e pedidos feitos para o rei. E, em cada fase, uma habilidade argumentativa diferente espera-se que seja ensinada.

A avaliação utilizada para a avaliação do jogo foi a completa. Os passos da avaliação, bem como os resultados são apresentados a seguir. O jogo foi avaliado por todas as suas fases implementadas. A avaliação começa durante a interação com o jogo, onde o avaliador "joga o jogo". Em seguida, a lista completa de técnicas mapeadas foi analisada. Uma a uma, as técnicas foram selecionadas ou descartadas caso estivessem ou não presentes no jogo. A partir da lista de técnicas presentes foram identificadas as MBs as quais elas são relacionadas dentro da estrutura interna. As motivações básicas obtidas foram: Significado épico \& chamado, Desenvolvimento \& realização, Empoderamento \& feedback, Influência social \& pertencimento, Escassez \& impaciência e Imprevisibilidade \& curiosidade.

Na MB "Significado épico \& chamado" foram identificadas três técnicas: narrative, humanity hero e destiny child. Na MB "Desenvolvimento e realização" foram identificadas cinco técnicas: badges, progress bar, glowing choice, quest list, e step by step tutorial. Esta foi a MB com mais técnicas utilizadas no jogo. As técnicas da MB "Empoderamento \& feedback" foram: real time control, instant feedback, choice perception e meaningful choices. As técnicas identificadas no jogo que caracterizaram a MB "Influência social \& pertencimento" foram: mentorship e conformy anchor. A MB "Escassez \& impaciência" foi impactada pelas seguintes técnicas: options pacing e patient feedback. E, por fim, a MB "Impaciência \& curiosidade" apresentou apenas uma técnica: visual storytelling.

Como resultado da avaliação simples do jogo, tem-se a seguinte conclusão: o jogo possui a presença de sete MBs, ele utiliza tanto aspectos relacionados às motivações intrínseca e extrínseca. Faz uso da motivação intrínseca quando aplica as técnicas de gamificação das motivações básicas: Desenvolvimento \& realização, Propriedade \& posse juntamente com Escassez \& impaciência. E da motivação intrínseca quando utiliza as MBs Empoderamento \& feedback, Influência social \& pertencimento e Imprevisibilidade \& curiosidade. A motivação extrínseca foca nos resultados do jogo e a intrínseca no processo do jogo. $\mathrm{O}$ jogo avaliado balanceou também as motivações positivas e negativas, prendendo o interesse do jogador, uma gamificação eficaz não é aquela que faz uso de todas as MBs,

\footnotetext{
${ }^{1}$ http://reed.cs.depaul.edu/peterh/Games/attk3/attk3.html
} 
V Congresso Brasileiro de Informática na Educação (CBIE 2016)

Anais do XXVII Simpósio Brasileiro de Informática na Educação (SBIE 2016)

mas sim quando consegue unir de maneira complementar o que produz motivação de forma homogênea.

Dando prosseguimento com a avaliação completa, foram utilizados os conhecimentos obtidos na etapa anterior: as MBs identificadas e a relação de técnicas identificadas. De seguida, os atributos das técnicas identificadas foram computados. Os valores que representam a relevância da técnica em relação ao atributo foram então calculados. $\mathrm{O}$ resultado, classificado do maior para o menor é apresentado na Tabela 1.

Tabela 1: Resultado da análise do jogo AttK.

\begin{tabular}{|l|c|}
\hline Atributo & Valor \\
\hline Participação & 2.708 \\
\hline Atenção & 2.344 \\
\hline Envolvimento com o trabalho & 2.302 \\
\hline Domínio & 2.167 \\
\hline Persistência & 1.844 \\
\hline Socialização & 1.690 \\
\hline
\end{tabular}

O atributo melhor avaliado foi a Participação, seguido da Atenção e do Envolvimento. Tais indicadores estão coerentes com a avaliação qualitativa do avaliador.

\section{Conclusões}

O uso da gamificação no ensino é aconselhável: (1) as novas gerações estão ambientadas com a dinâmica dos jogos, (2) os aspectos motivacionais da gamificação induzem os usuários a apenas jogarem, sem maiores comprometimentos e (3) a aprendizagem gamificada induz naturalmente o estudante a um aprender com prazer.

Entretanto a escolha do jogo a ser utilizado em sala de aula deve ser cuidadosamente avaliada pelo educador. Se por um lado existem jogos com conteúdos próprios para os diversos temas (matemática, história, geografia entre outros) e a avaliação da aderência do tema é de fácil avaliação pelo educador, por outro lado, não se encontram ferramentas e/ou metodologias que o auxiliem a identificar previamente o impacto esperado do jogo juntos aos estudantes.

Com este cenário em mente é fácil de identificar a contribuição da metodologia explanada e testada neste artigo: $\mathrm{O}$ educador pode agora antever quais serão as motivações básicas que influenciarão o jogador, bem como os atributos de engajamento que mais fortemente serão impactados pelo jogo. Este conjunto: aderência do tema e gamificação bem planejada têm sido utilizadas pelos autores deste trabalho e se mostrou extremamente útil na obtenção de resultados com a gamificação da sala de aula e de disciplinas inteiras.

Outro aspecto que não figura no texto, mas importa salientar é a facilidade da aplicação da metodologia. Neste momento, toda ela foi realizada através do uso de planilhas: (1) a construção da matriz de MBs, das técnicas de gamificação e do cruzamento os indicadores de engajamento com as técnicas, (2) a atribuição de notas aos indicadores pelos juízes e, por fim, (3) o teste do jogo AttK. 
V Congresso Brasileiro de Informática na Educação (CBIE 2016)

Anais do XXVII Simpósio Brasileiro de Informática na Educação (SBIE 2016)

O jogador pode ser influenciado por seu perfil do jogador e pelo contexto do jogo (Bartle, 1996). No entanto, a avaliação proposta neste trabalho teve como objetivo avaliar apenas o jogo e as suas motivações, não tendo sido considerada a influência das motivações e do contexto sobre os jogadores.

Por fim, como trabalhos futuros, identificaram-se a necessidade da extensão do número de atributos de engajamento, da pesquisa/utilização de outras características motivacionais para além do engajamento e aumento do número de juízes.

\section{Referências}

Appelo, J. (2014). Management 3.0 Workout: Games, Tools \& Practices to Engage People, Improve Work, and Delight Clients. Rotterdam: Happy Melly Express.

Bartle, R. (1996). Hearts, clubs, diamonds, spades: Players who suit muds. Journal of MUD research, 1 (1).

Borges, S. d., Reis, H. M., Durelli, V. H., Bittencourt, I. I., Jaques, P. A., \& Isotani, S. (2013). Gamificação Aplicada à Educação: Um Mapeamento Sistemático. Anais do XXIV Simpósio Brasileiro de Informática na Educação (SBIE 2013), (pp. 254-263).

Britt, A., Hastings, P., Durik, A., \& Kopp, K. (2009). Designing a game for teaching argumentation skills. AIED 2009: 14th International Conference on Artificial Intelligence in Education, Workshop on Intelligent Educational Games.

Chou, Y.-k. (2015). Actionable Gamification: Beyond Points, Badges, and Leaderboards. Leanpub.

Figueiredo, M., Paz, T., \& Junqueira, E. (2015). Gamificação e educação: um estado da arte das pesquisas realizadas no Brasil. Anais dos Workshops do IV Congresso Brasileiro de Informática na Educação (CBIE 2015), (pp. 1154 - 1163).

Fredricks, J. A., Blumenfeld, P. C., \& Paris, A. H. (2004). School Engagement: Potential of the Concept, State of the Evidence. Review of Educational Research, 74, pp. 59-109.

Freitas, S. A., Silva, W. C., \& Marsicano, G. (2016). Using an Active Learning Environment to Increase Students' Engagement. 29th Conference on Software Engineering Education and Training (pp. 232-236). Dallas - TX: IEEE.

Kapp, K. M., Blair, L., \& Mesch, R. (2014). The Gamification of Learning and Instruction Fieldbook: San Francisco - CA: John Wiley \& Sons, Inc.

Kumar, J. M., \& Herger, M. (2013). Gamification at Work: Designing Engaging Business Software. Interaction Design Foundation.

McGonigal, J. (2011). Reality Is Broken: Why Games Make Us Better and How They Can Change the World. Penguin Books.

Prensky, M. (2007). Digital Game-Based Learning. St. Paul - MN, USA: Paragon House.

Seixas, L. d., Gomes, A. S., Filho, I. J., \& Rodrigues, R. L. (2014). Gamificação como Estratégia no Engajamento de Estudantes do Ensino Fundamental. Anais do XXV Simpósio Brasileiro de Informática na Educação (SBIE 2014). 